\title{
Overview of laser-driven generation of electron-positron beams
}

\author{
G. Sarri ${ }^{1} \dagger$, M. E. Dieckmann ${ }^{2}$, I. Kourakis ${ }^{1}$, A. Di Piazza ${ }^{3}$, B. Reville ${ }^{1}$, \\ C. H. Keitel ${ }^{3}$ and M. Zepf ${ }^{1,4}$ \\ ${ }^{1}$ Centre for Plasma Physics, School of Mathematics and Physics, Queen's University of Belfast, \\ BT7 1NN, Belfast, UK \\ ${ }^{2}$ Department of Science and Technology (ITN), Linkoping University, 60174 Norrköping, Sweden \\ ${ }^{3}$ Max-Planck-Institut für Kernphysik, Saupfercheckweg 1, 69117 Heidelberg, Germany \\ ${ }^{4}$ Helmholtz Institute Jena, Frobelsteig 3, 07743 Jena, Germany
}

(Received 3 December 2014; revised 31 March 2015; accepted 1 April 2015;

first published online 19 May 2015)

Electron-positron $(e-p)$ plasmas are widely thought to be emitted, in the form of ultra-relativistic winds or collimated jets, by some of the most energetic or powerful objects in the Universe, such as black-holes, pulsars, and quasars. These phenomena represent an unmatched astrophysical laboratory to test physics at its limit and, given their immense distance from Earth (some even farther than several billion light years), they also provide a unique window on the very early stages of our Universe. However, due to such gigantic distances, their properties are only inferred from the indirect interpretation of their radiative signatures and from matching numerical models: their generation mechanism and dynamics still pose complicated enigmas to the scientific community. Small-scale reproductions in the laboratory would represent a fundamental step towards a deeper understanding of this exotic state of matter. Here we present recent experimental results concerning the laser-driven production of ultra-relativistic $e-p$ beams. In particular, we focus on the possibility of generating beams that present charge neutrality and that allow for collective effects in their dynamics, necessary ingredients for the testing pair-plasma physics in the laboratory. A brief discussion of the analytical and numerical modelling of the dynamics of these plasmas is also presented in order to provide a summary of the novel plasma physics that can be accessed with these objects. Finally, general considerations on the scalability of laboratory plasmas up to astrophysical scenarios are given.

\section{Introduction}

Pair-plasmas, i.e. plasmas consisting of negatively and positively charged particles bearing the same mass and (absolute) charge, have been gathering increasing interest among plasma researchers in the last years. Magnetized $e-p$ plasmas are predicted to exist in pulsar magnetospheres (Ginzburg 1971; Sturrock 1971; Ruderman and Sutherland 1975; Manchester and Taylor 1977; Michel 1982, 1991), in bipolar outflows (jets) in active galactic nuclei (AGN, Begelman et al. 1984; Miller and Witta 1987), at the centre of our own galaxy (Burns 1983), in the early universe (Gibbons et al. 1983), and in inertial confinement fusion schemes using ultraintense lasers (Liang et al. 1998; Gahn et al. 2000). Non-relativistic pair plasmas have also been created in experiments

$\dagger$ Email address for correspondence: g.sarri@qub.ac.uk 
(Greaves et al. 1994; Greaves and Surko 1995; Zhao et al. 1996) and, recently, Helander and Ward (2003) discussed the possibility of pair production in large tokamaks due to collisions between multi-MeV runaway electrons and thermal particles. Remarkably, (Oohara and Hatakeyama 2003; Hatakeyama and Oohara 2005; Oohara et al. 2005) plasmas composed of (two populations of) fully ionized particles with approximately the same mass and absolute charges of opposite polarity $(+/-)$, have recently been created in the laboratory (Oohara and Hatakeyama 2003) by creating a large ensemble of fullerene ions $\left(C_{60}^{+}\right.$and $C_{60}^{-}$, in equal numbers), thus allowing for a study of the properties of pair plasmas with no concern for mutual annihilation (recombination), which limits the lifetime of $e-p$ plasmas. Theoretical proposals for prolific production of pair plasmas during the collision of ultraintense laser beams have also been put forward (see Di Piazza et al. (2012) and references therein), which might become realistic with the advent of the next generation of laser facilities.

As mentioned above, the magnetospheres of rotating compact astrophysical objects are generally thought to be filled with an $e-p$ plasma, produced in pair cascades in the intense fields near the surface of the compact object. Although the exact details of how and where these cascades are occurring is incomplete, it is evident that the resulting pair plasma might play a central role in the dynamics of these objects, since the pairs produced ultimately populate any outflow, and the currents they carry inevitably determine the electromagnetic (EM) structure. Pulsars are perhaps the best known example, where $e-p$ pair production is an essential ingredient of global models (Kennel and Coroniti 1984). Magnetic fields at the surface of a pulsar are typically very strong, and can in some cases reach a significant fraction of the critical field strength $B_{\text {crit }}=m^{2} c^{3} / e \hbar=4.414 \times 10^{13} \mathrm{G}$. These fields rotate with the pulsar and pair production can proceed by a number of channels in such intense electric and magnetic fields. The standard picture assumes an initial particle is accelerated in either the rotational or space charge limited electric field, and emits a photon, that initiates a pair cascade (Ruderman and Sutherland 1975; Arons and Scharlemann 1979; Levinson et al. 2005; Reville and Kirk 2010; Timokhin and Arons 2013). The magnetic field strength is typically strong enough to ensure that the resulting pair plasma co-rotates with the pulsar. Beyond some critical point, co-rotation must cease, since it would require superluminal motion. To avoid this, the particles escape along open field lines, in the form of a relativistic pair wind. The details of how energy is dissipated in these winds is an active area of research (Kirk et al. 2009). A similar mechanism is thought to operate around rotating black holes, with an external magnetic field threading an accretion disc. It has also been suggested that the launching of jets from a rotating black-hole might rely on copious pair production near the black hole ergosphere, sufficient to permit force-free conditions in the magnetosphere. Blandford and Znajek (1977) have shown that, under these conditions, energy and angular momentum can be extracted electromagnetically, consistently with observations of AGN. These theoretical predictions are now well supported by three-dimensional magneto-hydrodynamics simulations including the effects of general relativity (Komissarov and Barkov 2009; Sadowski et al. 2014). As with the pulsar wind, the resulting outflow is pair dominated, until a significant amount of the ambient matter has been entrained. A similar mechanism is thought to be responsible for the launching of gamma-ray burst (GRB) jets, however, significant pair-production can also occur in the optically thick outflow, in these energetic events (Meszaros and Rees 1992).

The above phenomena, namely pulsar winds, AGN jets and GRBs, represent some of the most extreme environments in the Universe, with plasma conditions that are 
unattainable in terrestrial laboratories. In fact, producing a genuine pair plasma in the laboratory at all has proved to be extremely challenging experimentally. By exploiting recent advances in laser plasma acceleration, it is possible now to initiate a pair cascade in a high- $Z$ metal target, using a laser-wakefield accelerated ultrarelativistic electron beam. In this article, we will discuss recent results concerning laser-driven generation of $e-p$ beams, with particular attention to the possibility of generating neutral beams that allow for collective effects in their dynamics. This would open a new branch of experimental physics, namely the study of pair plasmas in the laboratory, which will be applicable to our understanding of the dynamics of ultra-massive astrophysical objects.

In Sec. 2, the theory behind the quantum EM cascade initiated by an ultrarelativistic electron in a high- $Z$ material is summarized. In Secs 3 and 4, we will discuss recent results reported in the literature concerning positron production either via direct laser irradiation of a solid target or via laser-wakefield accelerated electrons, respectively. Section 5 will summarize recent theoretical results on solitary waves in $e-p$ plasma, whereas Sec. 6 will discuss numerical work on the interaction with these plasmas with background electron-ion plasma. Section 7 will show general considerations on how to rescale laboratory results to astrophysical conditions and conclusive remarks will be given in Sec. 8 .

\section{Theory of quantum electromagnetic cascades in solids driven by an electron beam}

The propagation of an ultra-relativistic electron beam through a high- $Z$ solid target triggers the onset of a quantum EM cascade that might comprise three main steps: generation of a real high-energy photon via bremsstrahlung (Koch and Motz 1959), production of an $e-p$ pair during the propagation of a high-energy photon in the field of a nucleus (sometimes referred to as the Bethe-Heitler process) (Heitler 1954), and direct production of an $e-p$ pair mediated by the emission of a virtual photon during the propagation of an electron through the field of a nucleus (Baier and Katkov 2008). Hereafter, we will focus our attention on positron production as a result of $E M$ cascades in high- $Z$ targets in the ultra-relativistic regime. We can thus, neglect additional electron and positron energy losses as resulting, for instance, from additional scattering mechanisms (such as Compton, Möller, and Bhabha scattering) and from the ionization of the atoms themselves. In the following discussion, we will also adopt units whereby the electron mass $m_{\mathrm{e}}$, speed of light $c$, and the Planck's constant $h$ are all equal to 1 .

For sufficiently thick targets, we expect the newly generated electrons and positrons to be the seed for an additional chain of reactions, effectively inducing a multi-step cascade. In order to quantify this phenomenon, it is useful to rescale the target thickness $d$ in terms of the radiation length of the material $\left(L_{\mathrm{rad}}\right)$ (Tsai 1974). For an order-of magnitude estimate of $L_{\mathrm{rad}}$, we can assume here to be in the total-screening regime which, for an electron with energy $\varepsilon$ emitting a photon with energy $\omega$, occurs if the parameter $S \equiv \alpha Z^{1 / 3} \varepsilon(\varepsilon-\omega) /(\omega)$ is much larger than unity (here, $\alpha \approx 1 / 137$ is the fine structure constant and a Thomas-Fermi model of the atom is assumed (Beresteskii et al. 2008)). In this regime, and by including Coulomb corrections, the radiation length is approximately given by Beresteskii et al. (2008):

$$
L_{\mathrm{rad}} \approx 1 /\left[4 \alpha(Z \alpha)^{2} n \lambda_{\mathrm{C}}^{2} L_{0}\right]
$$


where $n$ is the number of atoms per unit volume, $\lambda_{\mathrm{C}}=3.9 \times 10^{-11} \mathrm{~cm}$ is the Compton wavelength, and $L_{0}=\log \left(183 Z^{-1 / 3}\right)-f(Z \alpha)$, with $f(x)=\sum_{a=1}^{\infty} x^{2} / a\left(a^{2}+x^{2}\right)$. Intuitively, a radiation length can be seen as the average distance needed to perform one step of the cascade (either photon creation by bremsstrahlung or generation of an $e-p$ pair). For example, the radiation length for $\mathrm{Pb}, \mathrm{Ta}$, and $\mathrm{Au}$ are $L_{\mathrm{rad}}^{\mathrm{Pb}} \approx 5.6$ $\mathrm{mm}, L_{\mathrm{rad}}^{\mathrm{Ta}} \approx 4.1 \mathrm{~mm}$, and $L_{\mathrm{rad}}^{\mathrm{Au}} \approx 3.3 \mathrm{~mm}$, respectively. It is thus convenient to express the target thickness in units of the material's radiation length $\ell=d / L_{\text {rad }}$.

$e-p$ production via virtual photon (a process sometimes called direct electroproduction (Heitler 1954)) is the dominant mechanism only for very thin targets $(\ell \ll 1)$ and we will therefore neglect its contribution in the rest of the manuscript. We then have only to consider bremsstrahlung and the Bethe-Heitler process, and it is straightforward to see that, in this case, the electron and positron distribution functions ( $f_{-}$and $f_{+}$, respectively) will have the same shape (Rossi 1952)

$$
\begin{aligned}
\frac{\partial f_{ \pm}}{\partial \ell}= & -\int_{0}^{1} d v \psi_{\mathrm{rad}}(v)\left[f_{ \pm}(E, \ell)-\frac{1}{1-v} f_{ \pm}\left(\frac{E}{1-v}, \ell\right)\right] \\
& +\int_{0}^{1} \frac{d v}{v} \psi_{\text {pair }}(v) f_{\gamma}\left(\frac{E}{v}, \ell\right),
\end{aligned}
$$

whereas the photon distribution function $\left(f_{\gamma}\right)$ can be written as

$$
\frac{\partial f_{\gamma}}{\partial \ell}=\int_{0}^{1} \frac{d v}{v} \psi_{\mathrm{rad}}(v)\left[f_{-}\left(\frac{E}{v}, \ell\right)+f_{+}\left(\frac{E}{v}, \ell\right)\right]-\mu_{0} f_{\gamma}(E, \ell),
$$

where we have defined

$$
\begin{gathered}
\psi_{\mathrm{rad}}(v)=\frac{1}{v}\left[1+(1-v)^{2}-(1-v)\left(\frac{2}{3}-2 b\right)\right], \\
\psi_{\text {pair }}(v)=v^{2}+(1-v)^{2}+v(1-v)\left(\frac{2}{3}-2 b\right),
\end{gathered}
$$

with $\mu_{0}=7 / 9-b / 3$ and $b=1 / 18 \log \left(183 / Z^{1 / 3}\right)$, are related to the cross section of bremsstrahlung and pair photo-production in the field of a heavy atom with charge number $Z$ (see Rossi (1952) for details).

As an example, we plot in Fig. 1 the amount of electrons and positrons escaping a solid target once a beam with $2.7 \times 10^{9}$ electrons with a flat spectrum ranging from 120 $\mathrm{MeV}$ to $1 \mathrm{GeV}$ enters a lead target of different thicknesses $\ell$. In a first approximation, we can see that the number of electrons decreases roughly exponentially, whereas the number of positrons initially increases and then starts to decrease exponentially as well. The latter behaviour is easily understood if we consider that, after a certain thickness, there is a non-negligible probability that also the newly generated positrons will start an EM cascade.

From this simple model we can, in a first approximation, assume that each electron/positron (photon) after a radiation length emits a photon (transforms into an $e-p$ pair) with half of the energy of the initial electron (with the electron and positron sharing half of the energy of the initial photon). Adopting this approximation, we can see that the maximum yield of positrons with an energy exceeding $E$ occurs for a target thickness $d_{\max } \sim L_{\mathrm{rad}} \log \left(\left\langle E_{\mathrm{e}}\right\rangle / E\right) / \log (2)$ (Rossi 1952), where $\left\langle E_{\mathrm{e}}\right\rangle$ is the average energy of the initial electron distribution. For the simple example discussed above, we have $\ell_{\max } \approx 2$. This can be intuitively understood if we consider that the electron will take on average one radiation length to generate a photon, which subsequently 


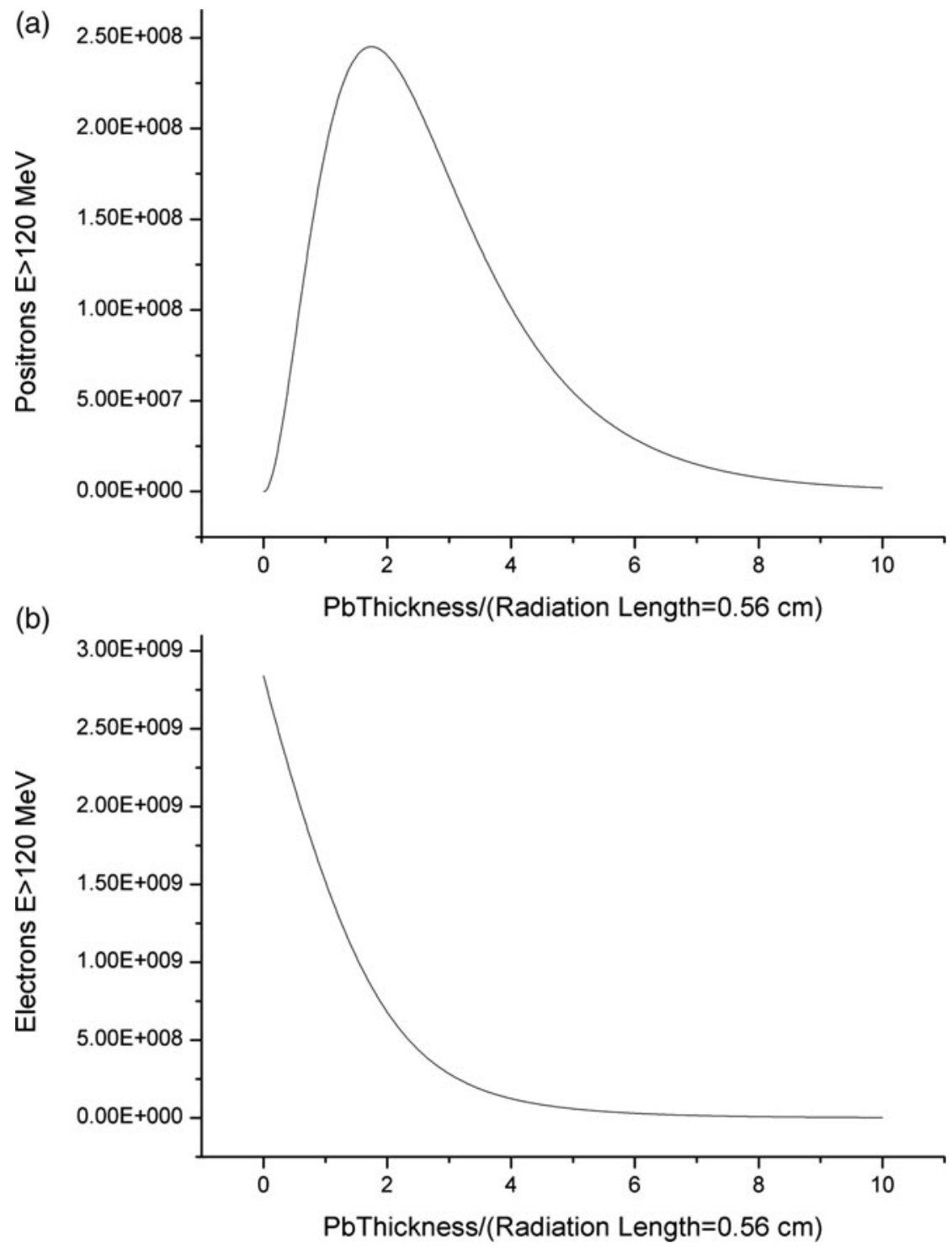

FiguRE 1. Number of positrons (a) and electrons (b) with energy exceeding $120 \mathrm{MeV}$ escaping a lead target of different thicknesses $\ell$ once an electron beam with a flat spectrum ranging from $120 \mathrm{MeV}$ to $1 \mathrm{GeV}$ (total number of electrons: $2.7 \times 10^{9}$ ) propagates through it. Image taken from Sarri et al. (2013a).

will need another radiation length to generate an $e-p$ pair. With this model, we see also that up to $\approx 2.5 \times 10^{8}$ relativistic positrons can be generated, i.e. approximately one-tenth of the initial electrons entering the solid target. It is worth pointing out that the number of positrons generated will roughly scale as: $N_{\mathrm{e}^{+}} \propto\left(Z^{2} n d\right)^{j}$, where $n$ is the number of atoms per unit volume in the material, $d$ is the thickness of the solid target, and $j \approx 1$ for the trident process and $j \approx 2$ for the Bethe-Heitler process (Sarri et al. 2013b, 2015).

Finally, the ultra-relativistic nature of the particles involved implies that the divergence of the electrons and positrons escaping the rear side of the target be 
roughly inversely proportional to their Lorentz factor. In the case of a thickness of exactly one radiation length, the cone angle will be $\left\langle\theta_{\mathrm{e}^{+}}^{2}\right\rangle \approx 1 / \gamma_{\mathrm{e}^{+}}^{2}$ (Rossi 1952) whereas for thicker target, a semi-empirical law can be given (Heitler 1954)

$$
\sqrt{\left\langle\theta_{\mathrm{e}^{+}}^{2}\right\rangle} \approx \frac{19.2}{p} \sqrt{\ell}
$$

with $p$ being the momentum of the particle in $\mathrm{MeV} \mathrm{c}^{-1}$. We still have an inverse proportionality with the particle Lorentz factor, with the only difference that the divergence increases also with the square root of the thickness of the material. Equation (2.6) is valid, however, only for a collimated electron beam. If the electron beam is already divergent (with cone-angle $\theta_{\mathrm{e}^{-}}$), the divergence of the escaping $e^{-}$ $p$ beam will instead be given by the geometrical sum of the two angles: $\left\langle\theta_{\text {real }}\right\rangle \approx$ $\sqrt{\left\langle\theta_{\mathrm{e}^{+}}\right\rangle^{2}+\left\langle\theta_{\mathrm{e}^{-}}\right\rangle^{2}}$.

As a final remark, it is important to note the consistency of these simple theoretical considerations with semi-empirical models extrapolated from experimental data in conventional accelerators upon which nuclear Monte-Carlo scattering codes such as GEANT4 and FLUKA (Battistoni et al. 2007) are largely based (see Yan et al. $(2013 a, b)$ for examples of modelling of these phenomena using these codes).

\section{Direct laser-solid irradiation}

The question then naturally arises: how can we generate an initial electron beam that we can then use to trigger the EM cascade inside a high- $Z$ solid target? In conventional particle accelerators, such as the $e-p$ collider (LEP), an electron beam is first accelerated by a few-meters long linear accelerator (LINAC). However, much more compact acceleration schemes have been identified in laser-plasma accelerators. The first intuitive approach is to focus a high-power laser onto the surface of a thin metallic foil. Without going into the details of the physics involved, we can just say that a laser beam with a non-perfect contrast (i.e. presenting a low-intensity pedestal before the arrival of the high-intensity peak of the pulse) will form a relatively cold pre-plasma on the irradiated surface of the target. This rather high-density plasma will be heated by inverse bremsstrahlung and its temperature can be approximated as (Langdon 1980):

$$
K_{\mathrm{B}} T_{\mathrm{e}} \approx 1.5\left(Z I_{\mathrm{L} 16}^{2} t_{10 \mathrm{fs}} / n_{\mathrm{e} 23}\right)^{2 / 9},
$$

where $K_{\mathrm{B}} T_{\mathrm{e}}$ is the electron temperature in $\mathrm{keV}, Z$ is the target atomic number, $n_{\mathrm{e} 23}$ is the electron density in units of $10^{23} \mathrm{~cm}^{-3}, I_{\mathrm{L} 16}$ is the laser intensity in units of $10^{16} \mathrm{~W} \mathrm{~cm}^{-2}$, and $t_{10 \mathrm{fs}}$ is the duration of the laser in units of $10 \mathrm{fs}$. The subsequent arrival of the high-intensity part of the laser will thus interact with this preformed plasma, generating, via $\overrightarrow{\mathbf{J}} \times \overrightarrow{\mathbf{B}}$ heating (sometimes also referred to as ponderomotive acceleration) (Kruer and Estabrook 1985), a divergent population of hot electrons with a characteristic temperature of

$$
\frac{K_{\mathrm{B}} T_{\mathrm{J} \times \mathrm{B}}}{m_{\mathrm{e}} c^{2}} \approx \sqrt{1+\frac{I_{\mathrm{L}} \lambda_{\mathrm{L}}^{2}}{2.8 \times 10^{18} \mathrm{Wcm}^{-2} \mu \mathrm{m}^{2}}}
$$

where $m_{\mathrm{e}} c^{2}$ is the electron rest energy, and $I_{\mathrm{L}}$ and $\lambda_{\mathrm{L}}$ are the laser intensity and central wavelength, respectively. If the laser polarization has a non-zero component parallel to the target normal, a resonant absorption mechanism must also be taken into account, producing electrons with a typical temperature given by Wilks and 
Kruer (1997)

$$
K_{\mathrm{B}} T_{\mathrm{res}} \approx 10\left(K_{\mathrm{B}} T_{\mathrm{e}} I_{\mathrm{L} 15} \lambda_{L \mu \mathrm{m}}\right)^{1 / 3},
$$

where $I_{\mathrm{L} 15}$ is the laser intensity in units of $10^{15} \mathrm{~W} \mathrm{~cm}^{-2}$ and $\lambda_{L \mu \mathrm{m}}$ is the laser central wavelength in micron.

If the target is sufficiently thick, we can use the same solid to both generate the electrons and to serve as a medium to sustain the EM cascade to generate the positrons. This approach was indeed adopted by Chen and collaborators in a series of experiments (Chen et al. 2009a,b, 2010, 2014) carried out using the Titan laser at the Jupiter laser facility at Lawrence Livermore National Laboratory and the OMEGA EP laser hosted by Rochester's Laboratory for Laser Energetics (Waxer et al. 2005). In both cases, the laser pulse duration was in the picosecond range, and the laser pulse energy varied from $100 \mathrm{~J}$ up to $850 \mathrm{~J}$ implying a peak laser power in the TW range. Fast-focusing optics ensured a laser focal spot of a few up to tens of microns, with an associated peak intensity of the order of $10^{19}-10^{20} \mathrm{~W} \mathrm{~cm}^{-2}$ onto the surface of mm-scale high- $Z$ solid targets. Assuming a ns pedestal with a contrast of $\approx 10^{-5}$ (Wilks and Kruer 1997; Chen et al. 2009a), we can estimate from (3.1) a temperature of the preformed plasma of the order of $20 \mathrm{keV}$ for $\mathrm{Au}$ and a slightly higher temperature for the hot electrons arising from resonance absorption. On the other hand, the $\overrightarrow{\mathbf{J}} \times \overrightarrow{\mathbf{B}}$ mechanism will give an estimated hot electron temperature of about $3 \mathrm{MeV}$ for a laser intensity of $10^{20} \mathrm{~W} \mathrm{~cm}^{-2}$. It is thus clear that this would be the dominant mechanism via which relativistic electrons of enough energy to generate an $e-p$ pair can be generated (electron energy at least twice the electron rest mass). However, it must be taken into account that nonlinear interactions of the intensity peak of the laser pulse with the preformed plasma can lead to a local enhancement of the laser intensity, especially via self-focussing effects (Sprangle et al. 1987), or to a self-modulated electron wakefield acceleration (Krall et al. 1993). These effects might explain why the recorded electron temperatures in this experiment were higher than those expected from a ponderomotive scaling (3.2).

By employing an $e-p$ magnetic spectrometer, an approximate number of $10^{11}$ electrons per steradian was obtained, in line with previous results on electron acceleration in laser-solid interactions (Gibbon et al. 2004). These electrons will present a wide divergence (of the order of tens of degrees), implying a typical electron density at the rear side of the solid target (assuming a thickness of $1 \mathrm{~mm}$ ) of the order of $10^{15}-10^{16} \mathrm{~cm}^{-3}$. The propagation of these electrons inside the solid target triggers an EM cascade whose main products are photons, electrons, and positrons. As intuitively expected, the positron yield is expected to increase with the target areal density and the target atomic number. In particular, the Bethe-Heitler process would imply a positron yield that scales with $Z^{4}$ and the square of the target areal density (Sarri et al. 2013b) explaining why a detectable positron signal was obtained only for the highest atomic number $(\mathrm{Au})$ used. In this case, the authors report on approximately $10^{8}$ positron $\mathrm{MeV}^{-1}$ steradian $^{-1}$ if a $1 \mathrm{~mm}$ thick $\mathrm{Au}$ target is used implying a conversion into positrons of the order of a few percent. It must be noted here that the radiation length for $\mathrm{Au}$ is of the order of $3.3 \mathrm{~mm}(\ell \approx 0.3)$ implying that both the Trident process (dominant for $\ell \leqslant 10^{-2}$ ) and higher order processes (significant only for $\ell>1$ ) can be neglected and only the Bethe-Heitler process is effectively responsible for the positron generation. In this case though, positron production is maximized for $\ell \approx 2$ (see Fig. 1) indicating that a higher positron yield could have been obtained if a thicker target had been used. 
The divergence of the positrons escaping the target will be given, in a first approximation, by the geometrical sum of the initial electron divergence $\left\langle\theta_{\mathrm{e}^{-}}\right\rangle$and the divergence associated with the positron generation $\left\langle\theta_{\mathrm{e}^{+}}\right\rangle$(see Sec. 2). Assuming a central positron energy of about $10 \mathrm{MeV},\left\langle\theta_{\mathrm{e}^{+}}^{2}\right\rangle \approx 1 / \gamma_{\mathrm{e}^{+}}^{2} \approx 3^{\circ}$, i.e. much smaller than the initial electron divergence (tens of degrees). The divergence of the escaping positron beam is thus dominated by the initial divergence of the electrons and it will be of the order of tens of degrees. In this configuration, we are thus in the presence of a broadly divergent and highly non-neutral $e-p$ population ( $\approx 5 \%$ of positrons) with a typical maximum density of the order of $10^{15}-10^{16} \mathrm{~cm}^{-3}$. Its relativistically corrected collisionless skin depth can be estimated to be of the order of $\ell_{\mathrm{e}} \approx 100-150 \mu \mathrm{m}$ implying that, in principle, collective behaviour of the $e-p$ population is possible, since the diameter of the beam is of the order of $D \approx 600 \mu \mathrm{m}\left(D>\ell_{\mathrm{e}}\right)$ and the longitudinal size of the beam is of the order of $L \approx 300 \mu \mathrm{m}>\ell_{\mathrm{e}}$ (assuming that the beam temporal duration is of the order of $1 \mathrm{ps}$, i.e. comparable to that of the laser beam). Nevertheless, this will be true only in the close proximity of the rear surface of the solid target. Moreover, the high-degree of non-neutrality makes it hard to appreciate the contribution of the positrons in the dynamics, which will virtually coincide with that of a purely electronic population.

It is also worth noticing that, without any additional plasma-like effects, the positrons are expected to exit the solid target with a spectral shape comparable to that of the electrons, i.e. Maxwellian. In an interesting series of experiments at higher laser energy, Chen and collaborators reported on the generation of quasimonoenergetic positron populations (Chen et al. 2010, 2014) with a maximum central energy of $19 \mathrm{MeV}$ and an energy spread of the order of $10-20 \%$. This improved positron spectrum will be indeed more appealing for further storage and acceleration, but it still far from a real applicability to laboratory astrophysics studies. The peaking of the positron spectrum can be attributed to a further acceleration at the rear of the target as induced by strong electrostatic (ES) fields set by the charge imbalance brought forward by the sheath of high-density electrons. This process, usually referred to as target normal sheath acceleration (Macchi et al. 2013), is indeed the dominant mechanism for laser-driven ion-acceleration at moderate laser intensities.

Despite the intrinsic interest of these experimental results, they do present limiting factors if an application to laboratory astrophysics is sought. In particular, it is difficult to achieve a neutral $e-p$ population (or, at least, a situation in which the positron contribution in the beam's dynamics is non-negligible). Moreover, the intrinsically high divergence obtained (of the order of tens of degrees, as dictated by the initial broad divergence of the electron generation mechanism) induces a quick drop in the particle density during propagation. It must be pointed out here that recent results obtained using the OMEGA EP laser facility suggest that subsequent magnetic collimation of the $e-p$ beam might slightly enhance the positron percentage of the beam, which is anyhow significantly lower than $50 \%$ (Chen et al. 2010). Besides, the rather demanding laser parameters required (energies in the $\mathrm{kJ}$ regime in a time duration of the order of picoseconds) make these results hardly scalable to higher energies.

\section{Laser-wakefield-accelerated electron beams propagating through a solid target}

The main limitations of the approach discussed in the last section can all be pinpointed to the relatively inefficient method used for the generation of a relativistic 
electron beam. The low energy and broad divergence of the positrons are indeed directly related to those of the electrons. It is clear that positron generation via EM cascade in a solid target will greatly benefit from a higher quality electron beam (narrower divergence and higher energy). Conventional particle accelerators are able to provide that, but stringent constraints on the stability of generated beams do not allow for an in-situ generation of $e-p$ beams via cascading. Indeed cascading is only used at an initial stage, in order to provide a low-energy positron population that can be stored in a storage ring and further accelerated (Potier and Rinolfi 1998). There are plans to use magnetic chicanes to recombine separate electron and positron beams (Muggli et al. 2013), but this configuration presents issues regarding ES stability of recombination. An alternative mechanism to ensure high-quality and high-energy electron beams is provided by laser-wakefield acceleration. Without going into the details of the scheme (see Esarey et al. (2009) for an up-to-date review on the subject), it will suffice here to say that electron beams with narrow divergence $(\approx \mathrm{mrad})$ and high energy (now exceeding $1 \mathrm{GeV}$ per electron) are now obtainable with widely available laser systems (laser energies of the order of a few Joules in pulse durations of the order of tens of femtoseconds) (Leemans et al. 2006, 2014; Wang et al. 2013).

We might then expect that a positron population with better qualities can be obtained if the electron beam acceleration stage (laser wakefield in a gas) and the electron/positron production stage (EM cascade in a solid) are physically separated. This approach was first adopted during a series of pioneering experiments by Gahn and collaborators (Gahn et al. 2000, 2002) whereby an ultra-short (duration of $130 \mathrm{fs}$ ) and low-energy laser pulse (energy of the order of $200 \mathrm{~mJ}$ ) was focussed at the edge of a high-density gas-jet with an electron density of the order of $0.3 n_{\mathrm{c}}$ where $n_{\mathrm{c}}$ represent the plasma critical density $\left(n_{\mathrm{c}} \approx 1.7 \times 10^{21} \mathrm{~cm}^{-3}\right.$ for $790 \mathrm{~nm}$ light $)$. In this regime, electrons are predominantly accelerated by direct laser acceleration (Pukhov et al. 1999). This mechanism is much less efficient than laser wakefield acceleration (Esarey et al. 2009), and only provides an electron population with a wide divergence and a Maxwellian energy distribution. The situation is thus comparable to electron acceleration in a solid, with the additional drawback that direct laser acceleration in gases generates an even lower number of electrons (approximately $10^{9}$ relativistic electrons per shot in Waxer et al. (2005)). However, the use of such a short laser pulse (duration of the order of $100 \mathrm{fs}$ ) ensures a much shorter electron population and therefore a much shorter positron bunch. In this series of experiments, the electrons generated by direct laser acceleration were then directed onto a $2 \mathrm{~mm}$ thick lead target $(\ell \approx 0.36)$ and direct evidence of the generation of positrons with an energy of the order of $2 \mathrm{MeV}$ was given. Again, the positron population represented only a few percent of the electron/positron cloud escaping the target, suggesting that the presence of positrons in the dynamics of the electron/positron cloud would be negligible.

It is thus clear that separating the electron acceleration stage (laser-gas interaction) from the positron generation stage (electron-solid interaction) became an appealing option only with the advent of the laser-wakefield acceleration. In this case, GeVlike electron beams with an overall charge of the order of $10 \mathrm{~s}$ of $\mathrm{pC}$ and a very narrow divergence (of the order of a few mrad) can be generated (see Leemans et al. (2006), (2014) and Wang et al. (2013) for recent experimental results in this area). Coupling a laser-wakefield accelerator with a solid target for the generation of positrons was first tested in a series of experiments at the HERCULES laser (Yanovsky et al. 2008) hosted by the Centre for Ultrafast Optical Science at the University of Michigan. In these experiments (Sarri et al. 2013a,b), a low-power laser 
beam ( $0.8 \mathrm{~J}$ in $30 \mathrm{fs}$ corresponding to a peak power of $27 \mathrm{TW})$ was focussed at the edge of a low-density gas-jet $\left(n_{\mathrm{e}} \approx 5 \times 10^{-3} n_{\mathrm{c}}\right)$ to generate, via ionization injection (Clayton et al. 2010), an electron beam with a broad spectrum peaking at $150 \mathrm{MeV}$, an overall charge of $50 \mathrm{pC}$, and a full width half-maximum divergence of $\theta_{\mathrm{e}^{-}}=1.4$ mrad. This electron beam was then directed onto a solid target of different material and thickness, producing a collimated $\left(\sqrt{\left\langle\theta_{\mathrm{e}^{+}}^{2}\right\rangle} \approx 3 \mathrm{mrad}\right.$ to be compared with $\approx 350$ $\mathrm{mrad}$ in the case of direct laser irradiation of the solid, see Sec. 3) and high-energy $\left(E_{\mathrm{e}^{+}} \leqslant 150 \mathrm{MeV}\right)$ positron beam with a maximum overall charge of 5-6 pC (i.e. $\approx$ $10 \%$ of the initial electron beam). The beam's divergence agrees with the expected theoretical value if we consider that these results were obtained for solids with a thickness comparable to the radiation length of each material $(\ell \leqslant 1)$. In this case, the positron beam divergence can be estimated as (see Sec. 2): $\left\langle\theta_{\text {real }}\right\rangle \approx \sqrt{\left\langle\theta_{\mathrm{e}^{+}}\right\rangle^{2}+\left\langle\theta_{\mathrm{e}^{-}}\right\rangle^{2}}=$ $\sqrt{1 / \gamma_{\mathrm{e}^{+}}^{2}+\left(\theta_{\mathrm{e}^{-}}\right)^{2}} \approx 3.6 \mathrm{mrad}$ for $\gamma_{\mathrm{e}^{+}} \approx 300$ (Sarri et al. 2013a), in good agreement with the experimental findings. Moreover, the positron yield is seen to scale with the square of the target thickness and the fourth power of the atomic number, as expected for a Bethe-Heitler process. Thanks to the low divergence and ultra-short beam duration $(\approx 30 \mathrm{fs})$, the positron density achieved in this experiment is $n_{\mathrm{e}^{+}}=2 \times 10^{14} \mathrm{~cm}^{-3}$. Despite the high-quality of the beam generated, Monte-Carlo simulations (using the code FLUKA (Battistoni et al. 2007)) indicate that positrons represent only $10 \%$ of the $e-p$ beam escaping the target indicating, once again, that the positrons will only have a negligible contribution to the dynamics of the electron/positron cloud.

In order to increase the number of positrons in the beam, two main paths can be pursued: we can either increase the thickness of the solid target, or we can increase the energy and charge of the initial electron beam. By direct comparison of Figs 1(a) and (b), we can also see that increasing the thickness of the solid target brings another interesting effect: for each material we can isolate a target thickness after which the ratio between the number of escaping electrons and positrons remains approximately constant. For $\mathrm{Pb}$, this appears to occur at approximately five radiation lengths in which the number of positrons and electrons with energy exceeding $120 \mathrm{MeV}$ is approximately $5 \times 10^{7}$ in both cases. This scenario was tested by Sarri and collaborators in a follow-up experiment carried out using the Astra-Gemini laser hosted by the Rutherford Appleton Laboratory (Hooker et al. 2006). The details of this experiment can be found in Sarri et al. (2015) and we here only focus on the main results.

By focussing a much more powerful laser beam (approximately $15 \mathrm{~J}$ in $45 \mathrm{fs}$, resulting in a peak power of the order of $300 \mathrm{TW}$ ) onto a low-density gas-jet, a broadband and energetic electron beam was accelerated following ionization injection (Clayton et al. 2010) (maximum energy of the order of $600 \mathrm{MeV}$, charge of $300 \mathrm{pC}$, and divergence of the order of a few mrad). This electron beam then propagated through a lead target of different thicknesses (from 1 to approximately 8 radiation lengths) producing a positron population of different density, spectrum, and divergence (see Sarri et al. (2013a,b)).

It is interesting to note that the maximum positron number was reached for approximately two radiation lengths (in agreement with the theoretical estimates shown in Sec. 2) inducing an estimated density of relativistic positrons of the order of $10^{17}$ positrons per centimetre cubed, i.e. comparable to what is achievable in conventional large-scale accelerators (see, for instance, the results obtained in SLAC (Ng et al. 2001; Blue et al. 2003)). For thicker targets, a regime is reached (approximately at 5 radiation lengths) whereby the number of escaping electrons and 
Gahn et al. (2002)

Chen et al. (2009a)

Chen et al. (2010)

Sarri et al. (2013b)

Sarri et al. (2015)

$$
E_{\mathrm{e}}^{+}
$$$$
\mathrm{N}_{\mathrm{e}}+\mathrm{MeV}^{-1} \mathrm{Sr}^{-1}
$$$$
\tau_{\mathrm{e}^{+}}
$$$$
\theta_{\mathrm{e}^{+}}
$$$$
0.13
$$$$
1-10
$$$$
1-10
$$$$
10^{10}
$$$$
5 \times 10^{3}
$$$$
3 \times 10^{5}
$$

$/$
350
350
3
20

$n_{\mathrm{e}^{+}}$
$1 \times 10^{14}$
$1 \times 10^{13}$
$2 \times 10^{14}$
$1 \times 10^{17}$

$$
\begin{gathered}
n_{\mathrm{e}^{+}} /\left(n_{\mathrm{e}^{-}}+n_{\mathrm{e}^{+}}\right) \\
/ \\
5 \%-10 \% \\
1 \% \\
1 \%-10 \% \\
0 \%-50 \%
\end{gathered}
$$

TABLE 1. Summary of the main parameters for the experiments reported in the literature concerning laser-driven positron generation. Please note that all the values listed are purely indicative and must serve only as an order-of-magnitude reference. $E_{\mathrm{e}^{+}}$represents the maximum recorded energy in $\mathrm{MeV}, N_{\mathrm{e}^{+}} \mathrm{MeV}^{-1} \mathrm{Sr}^{-1}$ gives an indication of the typical number of positrons per $\mathrm{MeV}$ per steradian, $\tau_{\mathrm{e}^{+}}$is the laser pulse duration in picoseconds, $\theta_{\mathrm{e}^{+}}$is the divergence of the high energy positrons in mrad, $\mathrm{n}_{\mathrm{e}^{+}}$is an estimated maximum positron density at the rear of the solid target in particles per centimetre cubed, and $n_{\mathrm{e}^{+}} /\left(n_{\mathrm{e}^{-}}+n_{\mathrm{e}^{+}}\right)$gives an indication of the percentage of positrons in the escaping electron-positron cloud.

positrons is approximately equal, indicating the generation of a neutral beam with a particle laboratory density of $2 \times 10^{16} \mathrm{~cm}^{-3}$ (related proper density of $1 \times 10^{15}$ $\left.\mathrm{cm}^{-3}\right)$. In this regime, the beam diameter is larger than the relativistic collision-less skin depth $\left(\ell_{\mathrm{e}} \approx c / \omega_{\mathrm{pe}}\right.$, where $\omega_{\mathrm{pe}}$ is the relativistically corrected plasma frequency). We are then in the presence of a neutral $e-p$ plasma, a unique state of matter to be produced in the laboratory that finally opens up the possibility for experimental studies with clear relevance to astrophysical scenarios (Sarri et al. 2015).

In conclusion, an indicative summary of the characteristics of the experimentally obtained laser-driven positron populations reported in the literature is given in Table 1, clearly elucidating the superior performance of schemes adopting laser wakefield acceleration for the generation of the primary electrons needed to initiate the EM cascade.

In the following two sections, a summary of the theoretical works reported in the literature describing peculiar characteristics of $e-p$ plasmas is given, with an emphasis on both the astrophysical scenarios in which they might play a role, and the experimental validations that can be provided.

\section{Theory for linear and nonlinear waves in pair (electron-positron) plasmas}

\subsection{Basics - physics of pair-plasmas and intrinsic linear modes}

From a theoretical point of view, $e-p$ plasmas are modelled as pair plasmas (p.p.), i.e. plasmas whose constituents are characterized by the same mass and (absolute) charge. The dynamics of such plasmas brings forward a number of new phenomena, some of which are rather unexpected. Contrary to the traditional textbook plasma picture, where distinct frequency scales are imposed by the mass difference between electrons and ions (Krall and Trivelpiece 1973; Stix 1992; Swanson 2003), the two pair species (i.e. electrons and positrons) respond on the same scale; in other words, the characteristic plasma frequencies for both components of opposite charge are identical (Stewart and Laing 1992; Iwamoto 1993; Zank and Greaves 1995). On the other hand, rather surprisingly, the dynamical characteristics of pair plasmas cannot always be inferred by simply taking the limit of equal (ion and electron) masses, formally.

Before presenting a summary of the main theoretical results reported in the literature, it is worth highlighting potential issues regarding fluid models, in particular 
TABLE I. Summary of electrostatic modes.

\begin{tabular}{cccc}
\hline \hline & $\theta=0$ & \multicolumn{2}{c}{$\theta=\pi / 2$} \\
Dispersion relation & Identification & Dispersion relation & Identification \\
\hline$\omega^{2}=C_{s}^{2} k^{2}$ & acoustic wave & $\omega=0$ & nonpropagating \\
$\omega^{2}=\Omega_{p}^{2}$ & cyclotron oscillation & $\omega^{2}=C_{s}^{2} k^{2}+\Omega_{p}^{2}$ & cyclotron waves \\
$\omega^{2}=2 \omega_{p}^{2}+\Omega_{p}^{2}$ & upper hybrid oscillation & $\omega^{2}=C_{s}^{2} k^{2}+2 \omega_{p}^{2}+\Omega_{p}^{2}$ & upper hybrid wave \\
$\omega^{2}=C_{s}^{2} k^{2}+2 \omega_{p}^{2}$ & Langmuir waves & $\omega^{2}=2 \omega_{p}^{2}$ & Langmuir oscillation \\
\hline \hline
\end{tabular}

FIGURE 2. Summary of fundamental electrostatic modes propagating in electron-positron plasmas. (Table reprinted from Zank and Greaves (1995).)

MHD, in highly collisionless systems. It is not immediately clear that the distribution function should be Maxwellian, nor is the pressure tensors guaranteed to be isotropic. In astrophysical systems, in which the Reynolds numbers are extremely large, and hence, the flows highly turbulent, it is not unreasonable to assume that scattering on magnetic fluctuations is sufficient to smooth out any large scale temperature anisotropy. Indeed, if the pressure is highly anisotropic, non-resonant mirror and firehose instabilities quickly drive the plasma back to a state of isotropy. Similar conclusions can be drawn in laboratory plasma situations.

Stewart and Laing (Stewart and Laing 1992) presented a study of normal p.p. modes via a multifluid description. Zank and Greaves (1995) have discussed the linear properties of various ES and EM modes in unmagnetized and in magnetized pair plasmas. The same authors have also considered two-stream instability and nonenvelope solitary wave solutions. Linear modes and associated instabilities in p.p. have also recently been revisited from a kinetic-theoretical point of view in Tsytovich and Wharton (1978) and Vranjes and Poedts (2005).

The fundamental dispersion profile of ES waves in e-p plasmas comprises an acoustic mode (subject to heavy Landau damping, in practice)

$$
\omega^{2}=C_{\mathrm{s}}^{2} k^{2}
$$

and a Langmuir-like mode

$$
\omega^{2}=\omega_{\mathrm{p}, \mathrm{e}}^{2}+\omega_{\mathrm{p}, \mathrm{p}}^{2}+C_{\mathrm{s}}^{2} k^{2}
$$

featuring a frequency gap in the infinite wavelength limit, viz. $\omega(k=0)=\sqrt{\omega_{\mathrm{p}, \mathrm{e}}^{2}+\omega_{\mathrm{p}, \mathrm{p}}^{2}}$ ( $=\sqrt{2} \omega_{\mathrm{p}}$ in symmetric plasmas) (Iwamoto 1993; Zank and Greaves 1995; Verheest and Cattaert 2004). In the latter expressions, $C_{\mathrm{S}} \simeq \frac{1}{\sqrt{2}}\left(\frac{T_{\mathrm{e}}}{m_{\mathrm{e}}}+\frac{T_{\mathrm{p}}}{m_{\mathrm{p}}}\right)^{1 / 2}$ represents the sound speed (Verheest and Cattaert 2004), $\omega_{\mathrm{p}, \mathrm{e}}=\left(\frac{n_{\mathrm{e}}, 0^{2}}{\epsilon_{\mathrm{p}} m_{\mathrm{e}}}\right)^{1 / 2}$ is the electron plasma frequency and $\omega_{\mathrm{p}, \mathrm{p}}=\left(\frac{n_{\mathrm{p}, 0} e^{2}}{\epsilon_{\mathrm{p}} m_{\mathrm{p}}}\right)^{1 / 2}$ is the positron plasma frequency (note that the latter coincide in symmetric $e-p$ plasmas, i.e. if $\left.n_{\mathrm{p}, 0}=n_{\mathrm{e}, 0}\right)$, while $\omega$ and $k$ are the wave frequency and wavenumber in the usual notation. In magnetized pair plasma, besides the ES upper-hybrid waves, one also encounters perpendicularly propagating ordinary and extraordinary modes as well as magnetic field-aligned EM waves, characterized in this case by linear polarization. Remarkably, no Faraday rotation occurs in pair plasmas. An elegant kinetic description of numerous linear collective modes in a non-relativistic pair magnetoplasma has been presented by Iwamoto (1993).

An exhaustive study of linear modes in both unmagnetized $(\mathbf{B}=\mathbf{0})$ and magnetized $(\mathbf{B} \neq \mathbf{0})$ cases can be found in Iwamoto (1993), and is here omitted for brevity. The main modes are summarized in Figures 2 and 3. Interestingly, an alternative approach to pair plasmas has been created in the laboratory by Hatakeyama and coworkers (Oohara and Hatakeyama 2003), who created a large ensemble of fullerene ions 
TABLE II. Summary of electromagnetic plasma modes. An asterisk (*) denotes a solution to Eq. (29).

\begin{tabular}{cccc}
\hline \hline & $\theta=0$ & & \multicolumn{2}{c}{$\theta=\pi / 2$} \\
Dispersion relation & Identification & Dispersion relation & Identification \\
\hline$\omega^{2}=C_{s}^{2} k^{2}$ & acoustic & $\omega=0$ & nonpropagating \\
$\omega=\omega_{\|+}$ & $R / L$ mode & $\omega=\omega_{\perp+}$ & extraordinary \\
$\omega=\omega_{\|-}$ & cyclotron & $\omega=\omega_{\perp-}$ & magnetosonic \\
$\omega=\omega_{1}^{*}$ & electron/cyclotron & $\omega=0$ & nonpropagating \\
$\omega=\omega_{2}{ }^{*}$ & $R / L$ mode & $\omega^{2}=c^{2} k^{2}+2 \omega_{p}^{2}$ & ordinary wave \\
$\omega=\omega_{3}^{*}$ & $\omega^{2}=C_{s}^{2} k^{2}+\Omega_{h}^{2}$ & upper hybrid wave \\
\hline \hline
\end{tabular}

FIGURE 3. Summary of fundamental electromagnetic modes propagating in electron-positron plasmas. (Table reprinted from Zank and Greaves (1995).)

$\left(C_{60}^{+}\right.$and $C_{60}^{-}$, in equal numbers). This system mimics $e-p$ plasmas and enables the study of their properties with no concern for mutual annihilation (recombination), which limits $e-p$ plasma lifetime in the lab. Those experimental investigations suggest the existence of three distinct modes (Oohara and Hatakeyama 2003). Two of these modes, namely an acoustic mode and a Langmuir-like high-frequency mode, are straightforward to predict via, e.g. two-fluid theory (Iwamoto 1993; Zank and Greaves 1995; Kourakis et al. 2006; Esfandyari-Kalejahi et al. 2006a,b), as discussed above. A third, intermediate-frequency mode was also reported (Oohara and Hatakeyama 2003), and it was viewed as a controversial topic by theoreticians: interpretations suggested include soliton-trains (Verheest and Cattaert 2004), ion acoustic wave acceleration by surplus electrons (Saleem et al. 2006) and Bernstein-Greene-Kruskal (BGK)-like trapped ion modes (Schamel and Luque 2005; Schamel 2008).

\subsection{Nonlinear effects}

Solitons. Nonlinear excitations (in the form of solitary waves or shocks) in pairplasmas have been studied quite extensively. Large amplitude structures have been modelled via the pseudo-potential approach (see e.g. in Verheest and Cattaert (2004) and references therein), and also by the associated Bernoulli quasi-fluid model (Verheest et al. 2004). Small-amplitude, weakly superacoustic pulses are modelled by the Korteweg-de Vries (KdV) formalism in the long wavelength limit (Hasegawa and Ohsawa 2004; Verheest and Cattaert 2004; Verheest 2006). In this widely used qualitative paradigm, the ES potential (disturbance) function $\phi$ (assumed to be weak) is shown to be governed by a nonlinear partial derivative equation (PDE) in the form (Verheest 2006 and Kourakis et al. 2009).

$$
\frac{\partial \phi}{\partial \tau}+A \phi \frac{\partial \phi}{\partial \xi}+B \frac{\partial^{3} \phi}{\partial \xi^{3}}=0,
$$

where $\xi=\epsilon^{1 / 2}\left(x-C_{\mathrm{s}} t\right)$ is a slow travelling wave space coordinate and $\tau=\epsilon^{3 / 2} t$ is a slow time variable. The resulting ES structure therefore is stationary in a frame moving at the speed of sound $C_{\mathrm{s}}$. Verheest (2006) has shown that the nonlinearity coefficient $A$ is related to the plasma parameters as $A \sim\left[1+\gamma_{\mathrm{e}}+\frac{T_{\mathrm{p}}}{T_{\mathrm{e}}}\left(1+\gamma_{\mathrm{p}}\right)\right]$, assuming polytropic indices $\gamma_{\mathrm{e} / \mathrm{i}}$ (viz., a pressure-density relation $p_{\mathrm{e}} \sim n_{\mathrm{e}}^{\gamma}$ for electrons, and similarly for positrons), hot (subsonic) electrons and 'cooler' (supersonic) positrons (in the opposite case, a permutation between subscripts $p$ and $e$ is imposed). Furthermore, the diffusion coefficient is $B \sim\left(1-\frac{T_{\mathrm{p}}}{T_{\mathrm{e}}}\right)^{2} /\left(1+\frac{T_{\mathrm{p}}}{T_{\mathrm{e}}}\right)$. $T_{e}$ and $T_{p}$ denote the temperature of the electron and positron component, respectively. 


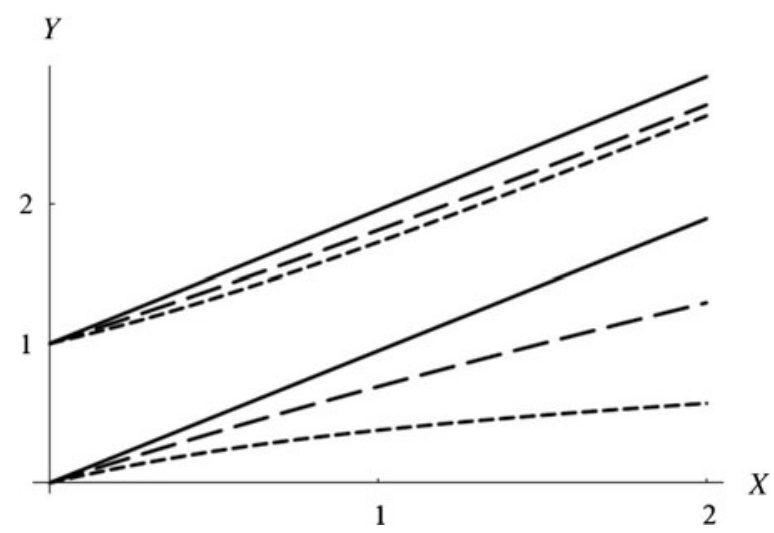

FIGURE 4. The dispersion curves for electrostatic modes in unmagnetized pair plasma $(\mathbf{B}=\mathbf{0})$ are shown. These include an acoustic mode (lower curves), which is in practice heavily damped (and does not exist in symmetric-temperature $e-p$ plasmas), and a Langmuir-type mode (upper curves). The curves correspond to values $T_{\mathrm{p}} / T_{\mathrm{e}}=0.1$ (small dashes), $T_{p} / T_{\mathrm{e}}=0.5$ (long dashes), and $T_{\mathrm{p}} / T_{\mathrm{e}}=0.9$ (full curves). The normalized variables in the horizontal and vertical axes are respectively $X=k^{2} T_{\mathrm{e}} /\left(m_{\mathrm{e}} \omega_{\mathrm{p}}^{2}\right)$ and $Y=\omega^{2} / \omega_{\mathrm{p}}^{2}$, where $\omega_{\mathrm{p}}^{2}=\omega_{\mathrm{p}, \mathrm{e}}^{2}+\omega_{\mathrm{p}, \mathrm{p}}^{2}$ is the sum of the frequency (square) of the pair species. Note that these curves are valid for $T_{\mathrm{e}}<T_{\mathrm{p}}$; on the other hand, if $T_{\mathrm{p}}>T_{\mathrm{e}}$, the same curves are obtained, but the subscripts $e$ and $p$ should be swapped for a correct interpretation of the above values. (Figure reprinted with permission from (Verheest 2006).)

The analytical expression for the bell-shaped (ES pulse soliton) solution reads

$$
\phi=\phi_{0} \operatorname{sech}^{2}\left(\frac{\xi-v t}{L}\right),
$$

where the solitonic pulse's maximum amplitude is $\phi_{0}=3 v / A$ and its width is $L=\sqrt{4 B / v}$. This expression defines a monoparametric family of solutions in terms of the real parameter $v$ (the soliton speed increment above the sound speed). Restoring the initial coordinates, it is straightforward to show that the total soliton speed is given by $v_{\text {sol }}=C_{\mathrm{s}}+\epsilon v$, suggesting a weakly supersonic propagation speed. Interestingly, it can be shown that there are no stationary-profile nonlinear structures in symmetric unmagnetized pair plasmas; in other words, pulse solitons may (only) be sustained by a temperature (or density) asymmetry between the pair species, and vanish in its absence (note that $B=0$ in the limit $T_{\mathrm{p}}=T_{\mathrm{e}}$ ). It can be seen that the same is actually true for the 'acoustic' mode (lower branch in Fig. 4), which is sustained by thermodynamic difference between the two pair species (and vanishes without it) (Verheest 2006).

Envelope solitons, modulational instability. Modulational instability (MI) and envelope solitons have also been studied, with respect to both ES (EsfandyariKalejahi et al. 2006a) and EM (Kourakis et al. 2007) modes. According to a generic picture (Kourakis and Shukla 2005), wavepackets can be modulated due to external effects (interaction with the turbulent plasma environment, wave-coupling, 'noise'), hence the amplitude, say $\psi$, becomes a modulated wave itself, encompassing the carrier wave $\exp [i(k x-\omega t)]$; in other words, the ES potential function reads $\phi \simeq \epsilon \psi \exp [i(k x-\omega t)]+\mathcal{O}\left(\epsilon^{2}\right)$ (omitting higher-order terms in higher harmonics). The wavepacket envelope $\psi$ is a slowly evolving function of space and time, which 
can be shown to obey a nonlinear PDE in the form (Esfandyari-Kalejahi et al. 2006a)

$$
i \frac{\partial \psi}{\partial T}+P \frac{\partial^{2} \psi}{\partial \zeta^{2}}+Q|\psi|^{2} \psi=0
$$

Here, $\zeta=\epsilon\left(x-v_{g} t\right)$ is a space variable moving at the group velocity $v_{\mathrm{g}}=\omega^{\prime}(k)$ (expressing a slow evolution in space, viz. $\epsilon \ll 1$ ), where $\omega=\omega(k)$ is the dispersion relation of the carrier, and $T=\epsilon^{2}$ is a 'slow' time variable. The dispersion coefficient $P$ is given by $\frac{1}{2} \frac{\partial^{2} \omega}{\partial k^{2}}$ (note that it vanishes for the acoustic mode in Fig. 1), while the nonlinearity coefficient is given by a lengthy expression of the temperature and number density ratios, $T_{\mathrm{p}} / T_{\mathrm{e}}$ and $n_{\mathrm{p}, 0} / n_{\mathrm{e}, 0}$, respectively (Esfandyari-Kalejahi et al. 2006a). The wavepacket may thus manifest a tendency for instability (MI) for carrierwavenumber values above a certain threshold (i.e. for short wavelengths): this is actually the root of $Q$, which can be shown to depend on the plasma configuration and intrinsic plasma values (see e.g. in Esfandyari-Kalejahi et al. (2006a)). A detailed analysis shows that energy localization may occur in the unstable (wavepacket) region, via the spontaneous formation of stable envelope structures (bright envelope solitons) (Kourakis and Shukla 2005), which mimic optical pulses in waveguides (or in optical fibers in long-distance communications).

The role of configurational asymmetry between the pair components (electronspositrons). Although symmetric pair components were originally assumed (i.e. at equal number density and temperature) in the modelling of p.p., it was soon realized that this picture may not be realistic. The remarkable features of symmetric and 'pure' pairplasmas may be modified in the presence of either temperature fluctuations (thermal mismatch between the two species), or density fluctuations creating a charge imbalance between the pair species. An overall charge imbalance may also be attributed to the presence of a 'third' species in the background. For instance, e-p plasmas may also contain positive ions, in addition to electrons and positrons. Electron-positron-ion $(e-p-i)$ plasmas are widely thought to occur in astrophysics, e.g. in AGN (Miller and Witta 1987), in pulsar magnetospheres (Michel 1982), and also in tokamaks and in the laboratory (Surko et al. 1986; Surko and Murphy 1990; Berezhiani et al. 1992; Greaves et al. 1994; Greaves and Surko 1995). E-p-i plasmas have also been studied, in this direction, with respect to low (ion-acoustic) (Salahuddin et al. 2002) and higher frequency (Esfandyari-Kalejahi et al. 2006a,b) ES modes. Magnetic field-aligned nonlinear Alfvén waves in an ultra-relativistic pair plasma have been investigated by Sakai and Kawata (1980) and Verheest (1996). Zhao et al. (1994) have performed three-dimensional EM particle simulations of nonlinear Alfvén waves in an $e-p$ magnetoplasma. EM p.p. wave modulation due to ponderomotive coupling to slow ES plasma perturbations was considered by Cattaert and collaborators (Cattaert et al. 2005).

Nonlinear profile of $e-p-i$ plasmas. The nonlinear dynamical profile of $e-p-i$ is rich. On the pair species vibration scale, nonlinear excitations predicted include solitons, either of ES (Verheest 2006; Lazarus et al. 2008; Kourakis et al. 2009) or EM (Verheest and Cattaert 2005) type, modelled e.g. by KdV type theories. A ZakharovKuznetsov model was also proposed, in higher dimensionality, for magnetized $e-p-i$ plasmas (Williams and Kourakis 2013). Furthermore, envelope solitons have been shown to occur on the high (pair-) frequency scale, as part of the evolution of either EM (Kourakis et al. 2007) or ES (Kourakis et al. 2006) wavepackets. On the other side of the scale, low-frequency oscillations of the massive background component (defects, or heavy ions) have been investigated via a Krylov-Bogoliubov-Mitropolsky 


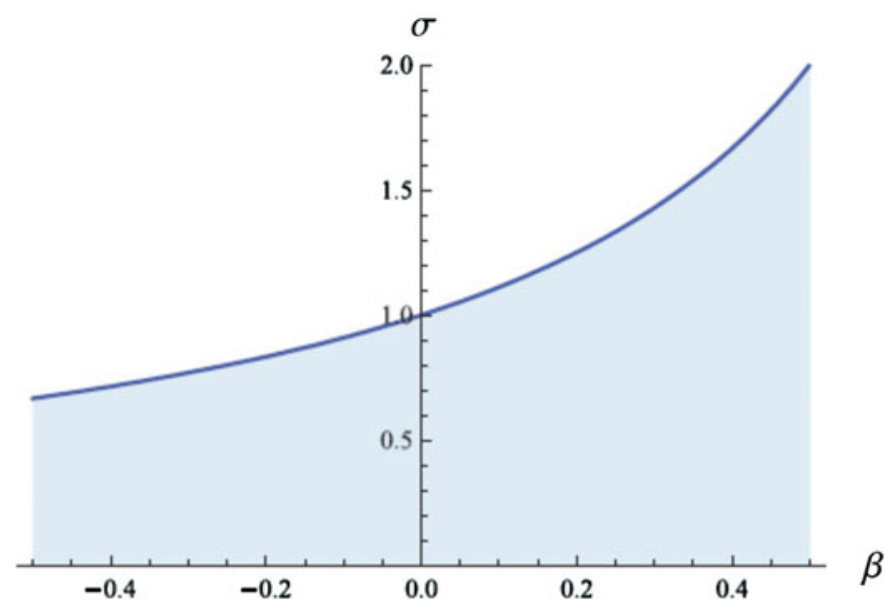

FIGURE 5. Soliton stability diagram for $e-p-i$ plasmas. The critical threshold of the temperature ratio $\sigma=T_{\mathrm{p}} / T_{\mathrm{e}}$ where the nonlinearity coefficient $A$ in (5.1) vanishes is shown against the (scaled) background ion density $\beta=Z_{\mathrm{i}} n_{\mathrm{i}} / n_{\mathrm{e}, 0}$. Note that $\beta=0$ represents 'pure' $e-p$ plasmas (no ions), while $\sigma=1$ represents symmetric-temperature $e-p-i$ plasmas. The shaded region below (above) the curve corresponds to parameter values where $A$ is positive (negative), i.e. where positive (negative) polarity potential pulses occur. (Figure reprinted with permission from Kourakis et al. (2009).)

perturbation technique, and were shown to be modulationally unstable at finite wavelengths (Salahuddin et al. 2002; Jehan et al. 2008).

Asymmetric pair-ion plasmas. It may be added, for completeness, that in an analogous qualitative picture (pair constituents, plus a heavier charged background species), one may anticipate the existence of a small fraction of charged massive particles in fullerene pair-ion plasma (Oohara and Hatakeyama 2003), either intrinsically as defects, or injected intentionally (doping) as a means of controlling plasma behaviour. Assuming the existence of a massive background species, of either positive or negative charge, the role of charged impurities (defects) in pair-plasmas was investigated e.g. in Kourakis and Saini (2010), where it was shown to affect the onset and characteristics (growth rate, wavenumber threshold) of MI of ES wavepackets. A temperature or/and density asymmetry may therefore act as an 'order' parameter in a way, and affect the characteristics and stability profile of linear waves and nonlinear structures (Kourakis et al. 2009); cf. Fig. 5.

\section{Numerical simulation of beam-plasma instabilities}

Plasma instabilities, which are driven by relativistic beams of leptons, have received widespread attention in an astrophysical context after observations confirmed that accreting compact objects (neutron stars or black holes) can emit relativistic plasma flows in the form of a jet. Examples for relativistic flows are pulsar winds and the jets of microquasars (Zhang 2013), AGN (Urry and Padovani 1995) and GRBs (Piran 2004). Plasma instabilities develop, when these relativistic outflows interact with an ambient plasma. This ambient plasma can be the stellar wind of the progenitor star that was emitted prior to the stellar collapse or the interstellar medium, like in the case of long GRBs. It can be the stellar wind of a companion star or the interstellar medium for microquasars or pulsar winds. The huge jets of AGN are slowed down by their interaction with the intergalactic medium. 
The plasma instabilities, which develop when a relativistically moving plasma interacts with an ambient medium, have been studied theoretically and by means of particle-in-cell (PIC) simulations (Dawson 1983). The bulk of the theoretical and simulation work has focused on instabilities in a spatially uniform plasma, which contains multiple beams of electrons or beams of electrons and positrons. The pioneering work concentrated on electron beam instabilities, which are driven by an unmagnetized relativistic beam of electrons with the number density $n_{\mathrm{b}}$ and a bulk electron population with a number density $n_{0}$. We will describe the three types of waves that are destabilized by such a beam. We go for this purpose into the reference frame defined by $n_{\mathrm{b}} \mathbf{v}_{\mathrm{b}}=-n_{0} \mathbf{v}_{0}$, where $\mathbf{v}_{0}$ is the mean speed of the bulk electrons. The ions are considered to be an immobile positively charged background. The net current vanishes in this frame of reference. The relativistic factor $\gamma$ of the beam electrons in the reference frame of the bulk electrons is practically equal to the Lorentz factor $\gamma_{\mathrm{b}}$ of $\mathbf{v}_{\mathrm{b}}$ if $n_{\mathrm{b}} \ll n_{0}$. The character of the instability depends on the angle between the wavevector of the unstable wave $\mathbf{k}$ and the beam velocity vector $\mathbf{v}_{\mathrm{b}}$.

The ES two-stream instability has a wavevector $\mathbf{k} \| \mathbf{v}_{\mathrm{b}}$. Its exponential growth rate scales with $\gamma_{\mathrm{b}}^{-1}$. This instability plays an important role for non-relativistic systems, but it is outrun by the other instabilities already at mildly relativistic values of $\mathbf{v}_{\mathrm{b}}$. A second instability, which is commonly referred to as the filamentation instability or beam-Weibel instability, has a wavevector $\mathbf{k} \perp \mathbf{v}_{\mathrm{b}}$. The filamentation instability yields the aperiodic growth of magnetic fields at an exponential growth rate $\propto \gamma_{\mathrm{b}}^{-1 / 2}$ if both counterstreaming electron beams have a similar density and temperature. Otherwise the instability becomes partially ES (Tzoufras et al. 2006). The oblique mode instability becomes important if $n_{0} \gg n_{\mathrm{b}}$. Its wavevector is oriented obliquely with respect to $\mathbf{v}_{\mathrm{b}}$ and the maximum value of its exponential growth rate scales as $\gamma_{\mathrm{b}}^{-1 / 3}$. This instability is almost ES. The scaling of the growth rates with $\gamma_{\mathrm{b}}$ has been verified in Dieckmann et al. (2006). Bret et al. (2008) has determined the range of parameters in which the individual instabilities dominate. Relativistic electron beam instabilities and their nonlinear saturation mechanisms have been reviewed in Bret et al. (2010). The effects of a magnetic field, which is aligned with $\mathbf{v}_{\mathrm{b}}$, has been examined in Bret et al. (2006). Lemoine and Pelletier (2011) and Lemoine et al. (2014) consider the effects of such instabilities on relativistic shocks and on particle acceleration.

Relativistic plasma outflows contain a sizeable fraction of positrons. The PIC simulations in Silva et al. (2003), Jaroschek et al. (2004), Jaroschek et al. (2005) and Sakai et al. (2000) were amongst the first to address the relativistic $e-p$ filamentation instability in three dimensions. The instabilities, which are driven by beams of pair plasma, cannot be distinguished from those of electron beams if no background magnetic field is present and if the wave amplitudes are small. This changes if baryons are present (Fiore et al. 2006) or if the wave amplitudes are no longer linear. The ES field, which is driven by the magnetic pressure gradient in the latter case, is weakened by the presence of positrons and the particles interact only via magnetic forces (Dieckmann et al. 2009).

More recent analytic and simulation work has focused on the formation and evolution of shocks in pair plasmas. Early PIC simulations of two $e-p$ clouds, which collided at a nonrelativistic speed, were performed in one (Hoshino et al. 1992) and in two dimensions (Kazimura et al. 1998). It was pointed out in Medvedev and Loeb (1999) that the magnetic fields, which are driven by the filamentation instability, give rise to the shock. That led to the suggestion that strong magnetic fields could be generated by this instability close to the internal shocks in the jets of GRBs (Brainerd 
2000; Medvedev and Loeb 1999), which triggered further PIC simulation studies of colliding pair plasmas e.g. those in Nishikawa et al. (2005), Milosavljevic and Nakar (2006), Milosavljevic et al. (2006) and Nishikawa et al. (2009). The effects of spatially uniform or striped magnetic fields in pulsar winds have been examined in a onedimensional PIC simulation (Nagata et al. 2008) and in two-dimensional simulations (Sironi and Spitkovsky 2009, 2011). A recent work has also studied the growth of the filamentation instability from noise levels to the formation of a full shock and the analytic estimate was confirmed by a PIC simulation (Bret et al. 2013, 2014).

Practically all PIC simulation studies have shown that if two lepton beams of comparable densities interact, then the filamentation instability dominates the plasma dynamics already at moderate relativistic factors of the collision speed or beam speed. The final state of this instability is composed of an ensemble of current flux tubes, which interact via collective EM forces. The flux tubes merge and the directed flow energy is gradually transferred to heat via reconnection processes (Polomarov et al. 2008). The currents are dissipated on electron time scales in a closed spatially uniform system. The currents are replenished if unperturbed plasma streams in, which happens close to shocks. Energetic leptons, which cross such a complex distribution of magnetic fields, experience a myriad of magnetic deflections and they radiate via the synchrotron jitter mechanism (Keenan and Medvedev 2013). The emitted photons are however not very energetic in most astrophysical jets due to relatively weak magnetic fields (Reville and Kirk 2010).

This previous work has assumed that the plasma is collisionless. This assumption is realistic for most astrophysical plasmas as far as binary interactions between plasma particles is concerned. However, the energetic leptons in relativistic jets will radiate and interact with photons. The emission of EM radiation cools off the lepton clouds. The scattering of particles by the photons is similar to binary collisions between particles. It will thus affect the filamentation instability by significantly modifying the distribution function of the particles, that might start to lose collective behaviour. In this respect, filamentation might even be suppressed, if scattering becomes more important than the collective interactions between particles. It thus remains unclear how such wave-particle collisions will affect the collective plasma dynamics under realistic conditions. This issue is now being addressed with PIC simulation studies (Lobet et al. 2014), but one has to keep in mind that such codes do not take into account all radiation emission and absorption mechanisms and that the PIC codes use approximations to describe these processes. Experimental studies of the interaction of relativistic lepton clouds thus remain the only means to test how robust the previous conclusions regarding lepton beam-driven instabilities and shocks are.

It has to be pointed out that the energy contained in a laser pulse is not yet sufficient to generate lepton clouds, which are large compared to the plasma scales of interest. The cloud dimensions would have to be of the order of hundreds of lepton skin depths in order to support a full leptonic shock, which is way beyond reach for contemporary lasers. The PIC simulation in Vieira et al. (2012) shows though that the lepton clouds generated in the laboratory may be large enough to trigger filamentation instabilities. Electron skin depth-sized lepton clouds actually favour the growth of the filamentation instability over the two-stream and oblique modes. Their wavevector is orthogonal to the mean velocity vector of the lepton cloud and the unstable spectrum of filamentation modes can extend to high wavenumbers if the leptons are sufficiently cool; the small wavelength of filamentation modes implies that they fit even into small lepton clouds. The resolution of the two-stream modes 
or the oblique modes would require a lepton cloud with a length along the cloud propagation direction that exceeds by far the electron skin depth.

\section{Scaling to astrophysical dimensions}

Despite the fact that the physical process of interest in astrophysical and laboratory systems generally operate on vastly different scales, both temporally and spatially, if both systems can be accurately modelled by a similar set of equations, it is generally possible to find a set of transformations that map one system onto the other. This process is relatively straightforward, and simply requires finding the relations between scaling coefficients of the dynamical variables i.e. $x \rightarrow \alpha x, t \rightarrow \beta t$ etc. that renders the governing system of equations invariant (Ryutov et al. 1999). The scaling relations for various fluid and kinetic plasma systems can be found in the literature: hydrodynamics (Ruytov et al. 1999), MHD (Ruytov et al. 2001; Cross et al.), radiation-MHD (Cross et al.; Ryutov et al. 2001), non-relativistic kinetic plasma (Ruytov et al. 2012; Connor and Taylor 1977).

Since the experiments reported in this paper are collisionless and relativistic, one could consider the full Vlasov equation together with Maxwell's equations. The resulting system is cumbersome, and provides little insight. Instead we take a simplified set of equations, appropriate for a cold relativistic plasma fluid. Assuming pair annihilation, and radiation reaction effects are negligible on the timescales of interest, the relevant equations for each species $\alpha$ are

$$
\begin{aligned}
& \frac{\partial n_{\alpha}}{\partial t}+\nabla \cdot n_{\alpha} \boldsymbol{v}_{\alpha}=0, \\
& \frac{d \boldsymbol{p}_{\alpha}}{d t}=q\left(\mathbf{E}+\frac{1}{c} \boldsymbol{v}_{\alpha} \times \boldsymbol{B}\right),
\end{aligned}
$$

where $n_{\alpha}$ is the lab-frame density, and $\boldsymbol{p}_{\alpha}=\gamma_{\alpha} m \boldsymbol{v}_{\alpha}$ is the cold fluid momentum of each species. Since we are ultimately interested in the growth of kinetic instabilities, the displacement current cannot be neglected, as has been done in previous nonrelativistic kinetic treatments (Ryutov et al. 1999; Connor and Taylor 1977). Our system of equations is closed by Maxwell's equations

$$
\begin{aligned}
& \nabla \times \boldsymbol{E}=-\frac{1}{c} \frac{\partial \mathbf{B}}{\partial t}, \\
& \nabla \times \boldsymbol{B}=\frac{1}{c} \frac{\partial \boldsymbol{E}}{\partial t}+\frac{4 \pi}{c} \sum_{\alpha} n_{\alpha} q_{\alpha} \boldsymbol{v}_{\alpha},
\end{aligned}
$$

where it is understood the summation is over all species of the plasma.

We seek a set of linear transformations for the varying quantities that leaves the above system of equations invariant. It is easily verified that the following transformation satisfies this condition:

$$
x \rightarrow a x ; t \rightarrow a t ; E \rightarrow b E ; B \rightarrow b B ; \gamma \rightarrow a b \gamma ; n \rightarrow(b / a) n,
$$

where $a$ and $b$ are dimensionless constants. Since our experimental beams are highly relativistic, it is customary to approximate the particle's velocities to be exactly $c$, and consider only the particle momentum: $p=\gamma m c$. It is clear from the scaling relations that the velocity is indeed invariant in all systems. In the context of high-intensity laser experiments, scaling from the laboratory to astrophysical systems, generally requires $a \gg 1$ (large systems and long temporal scales) and $b \ll 1$ (smaller fields, low densities). 
Finally we note that these scalings preserve the dimensionless quantity

$$
\varepsilon_{\mathrm{B}}=\frac{B^{2}}{4 \pi \gamma n m c^{2}}
$$

which, for a magnetic field direction perpendicular to the flow, is the ratio of energy flux carried by the EM field to that of the (cold) fluid. In our experiments, the time and length scales of interest are those associated with the (inverse) electron/positron plasma frequency and collision less skin-depth respectively.

$$
\omega_{\mathrm{pe}} t=\sqrt{\frac{4 \pi n e^{2}}{\gamma m}} t \text { and } \frac{x \omega_{\mathrm{pp}}}{c}=\sqrt{\frac{4 \pi n e^{2}}{\gamma m c^{2}}} x .
$$

These quantities are also preserved under the above transformations.

\section{Conclusions}

Recent experimental results concerning laser-driven production of $e-p$ beams have been discussed. Laboratory experiments are finally allowing for the generation of $e-p$ beams with present charge neutrality and plasma-like effects, thus providing an appealing experimental scenario for the study of key phenomena of pair plasma physics in the laboratory, which can then be scaled to astrophysical scenarios. A summary of the main analytical and numerical results in the field of pair plasma physics is also provided.

\section{Acknowledgements}

G.S. wishes to acknowledge support from EPSRC (Grant No.: EP/L013975/1). I.K. warmly acknowledges a number of inspiring discussions with Frank Verheest (Universiteit Gent, Belgium) and Manfred A. Hellberg (University of Kwazulu-Natal, Durban, South Africa) on topics included in the material presented herein.

\section{REFERENCES}

Arons, J. and Scharlemann, E. T. 1979 Astrophys. J. 231, 854.

Baier, V. N. and Katkov, V. M. 2008 Pisma Zh. Eksp. Teor. Fiz. 88(2), 88.

Battistoni, G. et al. 2007 AIP Conf. Proc. 896, 31.

Begelman, M. C., Blandford, R. D. and Rees, M. D. 1984 Rev. Mod. Phys. 56, 255.

Beresteskii, V. B., Lifshitz, E. M. and Pitaevskii, L. P. 2008 Quantum Electrodynamics. Oxford: Butterworth-Heinemann.

Berezhiani, V. I., Tskhakaya, D. D. and Shukla, P. K. 1992 Phys. Rev. A 46, 6608.

Blandford, R. D. and Znajek, R. L. 1977 MNRAS 179, 433.

Blue, B. E. et al. 2003 Phys. Rev. Lett. 90, 214801.

Brainerd, J. J. 2000 Astrophys. J. 538, 628.

Bret, A., Dieckmann, M. E. and Deutsch, C. 2006 Phys. Plasmas 13, 082109.

Bret, A., Gremillet, L., Benisti, D. and Lefebvre, E. 2008 Phys. Rev. Lett. 100, 205008.

Bret, A., Gremillet, L. and Dieckmann, M. E. 2010 Phys. Plasmas 17, 120501.

Bret, A., Stockem, A., Narayan, R. and Silva, L. O. 2014 Phys. Plasmas 21, 072301.

Bret, A. et al. 2013 Phys. Plasmas 20, 042102.

Burns, M. L. 1983 Positron-Electron Pairs in Astrophysics (ed. M. L. Burns, Harding, A. K. and R. Ramaty). New York: American Institute of Physics, pp. 281.

Cattaert, T., Kourakis, I. and Shukla, P. K. 2005 Phys. Plasmas 12(1), 012319.

Chen, H., Wilks, S., Bonlie, J., Liang, E., Myatt, J., Price, D., Meyerhofer, D. and Beiersdorfer, P. 2009a Phys. Rev. Lett. 102, 105001 
Chen, H. et al. 2009b Phys. Plasmas 16, 122702.

Chen, H. et al. 2010 Phys. Rev. Lett. 105, 015003.

Chen, H. et al. 2014 Phys. Plasmas 21, 040703.

Clayton, C. E. et al. 2010 Phys. Rev. Lett. 105, 105003.

Connor, J. W. and Taylor, J. B. 1977 Nuclear Fusion 17, 1047.

Cross, J. E., Reville, B. and Gregori, G. 2014 ApJ, 795, 59.

Dawson, J. M. 1983 Rev. Mod. Phys. 55, 403.

Dieckmann, M. E., Frederiksen, J. T., Bret, A. and Shukla, P. K. 2006 Phys. Plasmas 13, 112110.

Dieckmann, M. E., Shukla, P. K. and Stenflo, L. 2009 Plasma Phys. Control. Fusion 51, 065015.

Di Piazza, A., Müller, C., Hatsagortsyan, K. Z. and Keitel, C. H. 2012 Rev. Mod. Phys. 84, 1177.

Esarey, E., Schroeder, C. B. and Leemans, W. P. 2009 Rev. Mod. Phys. 81, 1229.

Esfandyari-Kalejahi, A., Kourakis, I., Mehdipoor M. and Shukla, P. K. 2006a J. Phys. A: Math. Gen. 39, 13817.

Esfandyari-Kalejahi, A., Kourakis, I. and Shukla, P. K. 2006b Phys. Plasmas 13, 122310/1-9.

Fiore, M., Silva, L. O., Ren, C., Tzoufras, M. A. and Mori, W. B. 2006 Mon. Not. R. Astron. Soc. 372, 1851.

Gahn, C., Tsakiris, G. D., Pretzler, G., Witte, K. J., Delfin, C., Wahlstrom, C.-G. and Habs, D. 2000Appl. Phys. Lett. 77, 2662.

Gahn, C., Tsakiris, G. D., Pretzler, G., Witte, K. J., Thirolf, P., Habs, D., Delfin, C. and Wahlstrom, C.-G. 2002 Phys. Plasmas 9, 987.

Gibbon, P., Beg, F. N., Clark, E. L., Evans, R. G. and Zepf, M. 2004 Phys. Plasmas 11, 4032.

Gibbons, G. W., Hawking, S. W. and Siklos, S. 1983 The Very Early Universe. Cambridge: Cambridge University Press.

Ginzburg, V. L. 1971 Sov. Phys. Usp. 14, 83

Greaves, R. G. and Surko, C. M. 1995 Phys. Rev. Lett. 75, 3846.

Greaves, R. G., Tinkle, M. D. and Surko, C. M. 1994 Phys. Plasmas 1, 1439.

Hasegawa, H. and Ohsawa, Y. J. 2004 Phys. Soc. Japan 73(7), 1764.

Hatakeyama, R. and Oohara, W. 2005 Phys. Scr. 116, 101.

Heitler, W. 1954 The Quantum Theory of Radiation. Oxford: Clarendon Press.

Helander, P. and Ward, D. J. 2003 Phys. Rev. Lett. 90, 135004.

Hooker, C. J. et al. 2006 J. Physique IV 133, 673.

Hoshino, M., Arons, J., Gallant, Y. A. and Langdon, A. B. 1992 Astrophys. J. 390, 454.

Iwamoto, N. 1993 Phys. Rev. E 47, 604.

Jaroschek, C. H., Lesch, H. and Treumann, R. A. 2004 Astrophys. J. 616, 1065.

Jaroschek, C. H., Lesch, H. and Treumann, R. A. 2005 Astrophys. J. 618, 822.

Jehan, N., Salahuddin, M., Saleem, H. and Mirza, A. M. 2008 Phys. Plasmas 15, 092301.

Kazimura, Y., Sakai, J. I., Neubert, T. and Bulanov, S. V. 1998 Astrophys. J. 498, L183.

Keenan, B. D. and Medvedev, M. V. 2013 Phys. Rev. E 88, 013103.

Kennel, C. F. and Coroniti, F. V. 1984 Astrophys. J. 283, 710.

Kirk, J. G., Lyubarsky, Y. and Petri, J. 2009 The Theory of Pulsar Winds and Nebulae, Astrophysics and Space Science Library, 357, 421-450.

Kirk, J. G. and Reville, B. 2010 Astrophys. J. 710, L16.

Koch, H. W. and Motz, J. 1959 Rev. Mod. Phys. 31, 920.

Komissarov, S. S. and Barkov, M. V. 2009 MNRAS 397, 1153.

Kourakis, I., Esfandyari-Kalejahi, A., Mehdipoor, M. and Shukla, P. K. 2006 Phys. Plasmas 13(5), 052117.

Kourakis, I., Moslem, W. M., Abdelsalam, U. M., Sabry, R. and Shukla, P. K. 2009 Plasma Fusion Res. 4, 018.

Kourakis, I. and Saini, N. S. 2010 J. Plasma Phys. 76(3-4), 607.

Kourakis, I. and Shukla, P. K. 2005 Nonlinear Process. Geophys. 12, 407.

Kourakis, I., Verheest, F. and Cramer, N. 2007 Phys. Plasmas 14(2), 022306.

Krall, J. et al. 1993 Phys. Rev. E 48, 2157.

Krall, N. A. and A. W. Trivelpiece 1973 Principles of Plasma Physics, New York: McGraw-Hill, pp. 9.

Kruer, W. L. and Estabrook, K. 1985 Phys. Fluids 28, 430. 
Langdon., A. B. 1980 Phys. Rev. Lett. 44, 575.

Lazarus, I. J., Bharuthram, R. and Hellberg, M. A. 2008 J. Plasma Phys. 74, 519.

Leemans, W. P. et al. 2006 Nature Phys. 2, 696.

Leemans, W. P. et al. 2014 Phys. Rev. Lett. 113, 245002.

Lemoine, M. and Pelletier, G. 2011 Mon. Not. R. Astron. Soc. 417, 1148.

Lemoine, M., Pelletier, G., Gremillet, L. and Plotnikov, I. 2014 Mon. Not. R. Astron. Soc. 440, 1365.

Levinson, A. et al. 2005 Astrophys. J. 631, 456.

Liang, E. P., Wilks, S. C. and Tabak, M. 1998 Phys. Rev. Lett. 81, 4887

Lobet, M., Ruyer, C., Debayle, A., d'Humières, E., Grech, M., Lemoine, M. and Gremillet, L. 2014 Phys. Rev. Lett., submitted.

Macchi, A., Borghesi, M. and Passoni, M. 2013 Rev. Mod. Phys. 85, 751.

Manchester, R. N. and Taylor, J. H. 1977 Pulsars. San Francisco: Freeman.

Medvedev, M. V. and Loeb, A. 1999 Astrophys. J. 526, 697.

Meszaros, P. and Rees, M. J. 1992 MNRAS 257, 29P.

Michel, F. C. 1982 Rev. Mod. Phys. 54, 1.

Michel, F. C. 1991 Theory of neutron Star Magnetospheres. Chicago: University of Chicago Press.

Pulsars: Problems and Progress (Astrophysical Society of the Pacific Conference Series 105), (ed. S. Johnston, M. A. Walker and M. Bailes) San Francisco: ASP, 1996.

Miller, H. R. and Witta, P. J. 1987 Active Galactic Nuclei. Berlin: Springer-Verlag, pp. 202.

Milosavljevic, M. and Nakar, E. 2006 Astrophys. J. 641, 978.

Milosavljevic, M., Nakar, E. and Spitkovsky, A. 2006 Astrophys. J. 637, 765.

Muggli, P. et al. 2013 ArXiv:1306.4380v1.

Nagata, K., Hoshino, M., Jaroschek, C. H. and Takabe, H. 2008 Astrophys. J. 680, 627.

Ng, J. S. T. et al. 2001 Phys. Rev. Lett. 87, 244801

Nishikawa, K. I., Hardee, P., Richardson, G., Preece, R., Sol, H. and Fishman, G. J. 2005 Astrophys. J. $622,927$.

Nishikawa, K. I. et al. 2009 Astrophys. J. 698, L10.

Oohara, W., Date, D. and Hatakeyama, R. 2005 Phys. Rev. Lett. 95, 175003.

Oohara, W. and Hatakeyama, R. 2003 Phys. Rev. Lett. 91, 205005.

Piran, T. 2004 Rev. Mod. Phys. 76, 1143.

Polomarov, O., Kaganovich, I. and Shvets, G. 2008 Phys. Rev. Lett. 101, 175001.

Potier, J. P. and Rinolfi, L. 1998 Proc. 6th European Particle Accelerator Conference, Stockholm, Sweden, pp. 859-861.

Pukhov, A., Sheng, Z.-M. and Meyer-ter-Vehn, J. 1999 Phys. Plasmas 6, 2847.

Reville, B. and Kirk, J. G. 2010 Astrophys. J. 715, 186.

Rossi, B. 1952 High-Energy Particles. New York: Prentice-Hall.

Ruderman, M. A. and Sutherland, P. G. 1975 Astrophys. J. 196, 51.

Ruderman, M. A. and Sutherland, P. G. 1975 Astrophys. J. 196, 51.

Ryutov, D. et al. 1999 Astrophys. J. 518, 821.

Ryutov, D. et al. 2001 Phys. Plasmas 8, 1804.

Ryutov D. et al. 2012 Plasma Phys. Control. Fusion 54, 105021.

Sadowski, A. et al. 2014 MNRAS 439, 503.

Sakai, J. and Kawata, T. J. 1980 Phys. Soc. Japan 49, 753.

Sakai, J., Nakayama, T., Kazimura, Y. and Bulanov, S. 2000 J. Phys. Soc. Japan 69, 2503.

Salahuddin, M., Saleem, H. and Saddiq, M. 2002 Phys. Rev. E 66, 036407.

Saleem, H., Vranjes, J. and Poedts, S. 2006 Phys. Lett. A 350, 375.

Sarri, G. et al. 2013a Plasma Phys. Control. Fusion 55, 124017.

Sarri, G. et al. 2013b Phys. Rev. Lett. 110, 255002.

Sarri, G. et al. 2015 Nat. Comm 6, 6747.

Schamel, H. 2008 J. Plasma Phys. 74, 725.

Schamel, H. and Luque, A. 2005 New J. Phys. 7, 69.

Silva, L. O., Fonseca, R. A., Tonge, J. W., Dawson, J. M., Mori, W. B. and Medvedev, M. V. 2003 Astrophys. J. 596, L121.

Sironi, L. and Spitkovsky, A. 2009 Astrophys. J. 698, 1523. 
Sironi, L. and Spitkovsky, A. 2011 Astrophys. J. 741, 39.

Sprangle, P. et al. 1987 IEEE Trans. Plasma. Sci. PS-15, 145.

Stewart, G. A. and Laing, E. W. 1992 J. Plasma Phys. 47, 295.

Stix, Th. 1992 Waves in Plasmas, New York: American Institute of Physics, pp. 6 and 26.

Sturrock, P. A. 1971 Astrophys. J. 164, 529.

Surko, C. M., Levelhal, M., Crane, W. S., Passne, A. and Wysocki, F. 1986 Rev. Sci. Instrum 57, 1862.

Surko, C.M. and Murphy, T. 1990 Phys. Fluid B 2, 1372.

Swanson, D. G. 2003 Plasma Waves, Bristol, UK: Institute of Physics, pp. 19.

Timokhin, A. N. and Arons, J. 2013 MNRAS 429, 20.

Tsai, Y. 1974 Rev. Mod. Phys. 46815.

Tsytovich, V. and Wharton, C. B. 1978 Commun. Plasma Phys. Control. Fusion 4, 91.

Tzoufras, M., Ren, C., Tsung, F. S., Tonge, J. W., Mori, W. B., Fiore, M., Fonseca, R. A., and Silva, L. O. 2006 Phys. Rev. Lett. 96, 105002.

Urry, C. M. and Padovani, P. 1995 Publ. Astron. Soc. Pac. 107, 715.

Verheest, F. 1996 Phys. Lett. A 213, 177.

Verheest, F. 2005 Nonlinear Proc. Geophys. 12, 569.

Verheest, F. 2006 Phys. Plasmas 13, 082301.

Verheest, F. and Cattaert, T. 2004 Phys. Plasmas 11, 3078.

Verheest, F., Cattaert, T., Lakhina, G. S. and Singh, S. V. 2004 J. Plasma Phys. 70(2), 237.

Vieira, J., Fang, Y., Mori, W. B., Silva, L. O. and Muggli, P. 2012 Phys. Plasmas 19, 063105.

Vranjes, J. and Poedts, S. 2005 Plasma Sources Sci. Technol. 14, 485.

Wang, X. et al. 2013 Nature Commun. 4, 1.

Waxer, L. J. et al. 2005 Opt. Photon. News 16, 30.

Wilks, S. C. and Kruer., L. 1997 IEEE J. Quantum Electron. 33, 1954.

Williams, G. and Kourakis, I. 2013 Phys. Plasmas 20, 122311.

Yan, Y. et al. 2013a Phys. Plasmas 20, 103106.

Yan, Y. et al. 2013b Phys. Plasmas 20, 103114.

Yanovsky, V. et al. 2008 Opt. Express 16, 2109.

Zank, G. P. and Greaves, R. G. 1995 Phys. Rev. E 51, 6079.

Zhang, S. N. 2013 Frontiers Phys. 8, 630.

Zhao, J., Nishikawa, K. and Sakai, J. I. 1994 Phys. Plasmas 1, 103.

Zhao, J., Sakai, J. I. and Nishikawa, K. 1996 Phys. Plasmas 3, 844. 